\title{
Study on Dispersion Stability of Carbon Nanotubes with Metal Coating
}

\author{
Xiao-ning LIANG $^{1, a}$, Wei LI ${ }^{2, b, c^{*}}$ \\ ${ }^{1}$ College of Textiles, Donghua University No. 1, Lane 300, Wenhui Rd, Songjiang university town, \\ Shanghai 201620, China \\ ${ }^{2}$ College of Textiles, Donghua University, Shanghai 201620, China \\ a1099006@mail.dhu.edu.cn, ${ }^{b}$ liwei@dhu.edu.cn, cwaiwentougao@outlook.com
}

Keywords: carbon nanotubes, multi-walled, dispersion, stability, metal-coated

Abstract. In this paper, the dispersion stability of multi-walled carbon nanotubes in combination with different metal particles was investigated. The available time of dispersions of metal-coated carbon nanotubes prepared by water bath ultrasonic dispersion was obtained. The UV-vis spectrophotometer was then adopted to measure the absorbance of the prepared carbon nanotubes dispersions. Under conditions of temperature control and no temperature control, the influences of different types of surfactants (SDBS, oleic acid, and polymers) on the stability of metal-coated carbon nanotubes dispersions were studied and compared with the results of untreated multi-walled carbon nanotubes. The results demonstrated enhanced stability of carbon nanotubes dispersions prepared by a custom temperature control system. The order of dispersants effects is polymers $>$ SDBS $>$ oleic acid, and the dispersion stability decreased in an order of untreated carbon nanotubes $>$ iron-coated carbon nanotubes $>$ nickel-coated carbon nanotubes. The metal coating increased the density of carbon nanotubes, thereby easily inducing settlement and reducing the dispersion suspension stability.

\section{Introduction}

Since carbon nanotubes possess excellent mechanical and electro and/or heat conductive properties [1-6], they have been extensively applied in modern composites field. In order to further improve the electro and heat conductivity, carbon nanotubes were composited with materials with high electro and/or heat conductivity such as nickel, iron and other metals [7,8]. Because of the particular structure of carbon nanotubes, the van der Waals force leads to inferior solubility in water or organic solvents, resulting in difficulties in preparation of stable carbon nanotubes dispersions.

The current dispersion methods primarily include mechanical dispersion [9], ultrasonic dispersion [10], chemical modification [11], dispersants [12], polymer coating method [13], and metal coating method [14], of which the chemical treatment and ultrasonic process will cause damage to the structure of carbon nanotubes $[15,16]$. For the purpose of better dispersion effects, a combination of multiple methods for dispersion has been accepted. Specifically, ultrasonic dispersion is conducted with carbon nanotubes in solutions to obtain dispersions with stable quality, which is then dispersed in resin matrix using mechanical mixture method to further ensure the quality of carbon nanotubes composites. Therefore, the stable dispersity of carbon nanotubes dispersion over a long period of time is a key part of carbon nanotubes composites manufacturing.

The heat energy generated in the ultrasonic process will accelerate molecular motions, which commonly causes secondary agglomeration of carbon nanotubes [17]. However, the addition of different nano-level metal particles will change the morphology of carbon nanotubes and its sensitivity to temperature. Meanwhile, the metals will significantly influence the density of carbon nanotubes, resulting in difficulties in control of the dispersion process and the dispersion stability.

In this study, the influence of temperature on the stability of multi-walled carbon nanotubes dispersion prepared in water/acetone solutions was studied. And comparisons of dispersion stability were made between carbon nanotubes with different metal coatings and untreated carbon nanotubes. The absorbance was measured using UV-vis spectrophotometer. The results showed that, by using a custom temperature control system, the dispersity of prepared dispersions was able to keep stable in a 
long period of time. The combination with metal particles decreased the stability of carbon nanotubes dispersions.

\section{Materials and Test}

Materials. The test includes untreated multi-walled carbon nanotubes, nickel-coated multi-walled carbon nanotubes, and iron-coated multi-walled carbon nanotubes, their properties are shown in Table 1 .

Table 1. Properties of untreated, nickel-coated and iron-coated carbon nanotubes

\begin{tabular}{cccc}
\hline & Tap density[g/cm3] & Outer diameter[nm] & length[um] \\
\hline 1\#untreated & 0.18 & $>50$ & $10-20$ \\
2\#nickel-coated & 0.83 & $>50$ & $10-20$ \\
3\#iron-coated & 0.2 & $2-50$ & $\sim 50$ \\
\hline
\end{tabular}

The dispersants employed were SDBS, oleic acid and polymers. The type of dispersant and the corresponding combined medium are listed in Table 2.

Table 2. Dispersants and corresponding combined medium

\begin{tabular}{ccc}
\hline Dispersants & Type & Combined medium \\
\hline oleic acid & anionic & Acetone \\
TNEDIS & nonionic/polymer & Acetone \\
SDBS & anionic & water \\
\hline
\end{tabular}

Preparation of dispersion samples. The carbon nanotubes solutions with a concentration of $0.5 \mathrm{vol} \%$ were prepared and received $120 \mathrm{~min}$ ultrasonic dispersion in a JAC-2010P ultrasonic cleaner. The dispersions were obtained after defoaming.

Dispersion test. The carbon nanotubes dispersions were kept static, and then the top layer solutions were extracted as samples. The sample absorbance was then measured at wavelength of $260.5 \mathrm{~nm}$ using UV-2550 UV-vis spectrophotometer.

In the test, the absorbance is proportional to the particles number within unit volume solution [18], so the dispersion degree of carbon nanotubes in the solution could be analyzed based on the absorbance value. The dispersity mainly includes: particle size, uniformity of particles in the solution, the settlement property of particles (stability of the dispersion).

Generally, small particles tend to suspend in the top of the solution, while large/heavy particles will fall to the bottom. The solid particles bear both the supporting force of dispersants and the gravity. Heavy particles are easily setting, while big forces between dispersants will hinder the settlement.

\section{Results and Discussion}

Relationship between static duration and absorbance. Fig. 1 shows the variations of absorbance for three carbon nanotubes SDBS/water dispersion samples with increasing static duration. It can be seen that the three curves have similar shapes, namely, rapidly decreasing in the initial stage, decreasing at a lower speed as time goes up, and finally stablizing at a smooth level. The absorbance of untreated carbon nanotubes showed a smooth decrease with increasing time, reaching a stable condition after 4 hours. The absorbance of nickel-coated carbon nanotubes decreased dramatically in the initial period without fluctuation. During the smooth decrease period after 4 hours, the decrease speed was higher than that of untreated carbon nanotubes. The absorbance of iron-coated carbon nanotubes fluctuated initially, with a decreasing speed between those of untreated and nickel-coated carbon nanotubes. The results are mainly due to the increased density of carbon nanotubes as a result of metal coating, 
resulting in smaller aperture, and, thus, easy settlement. Meanwhile, the metal particles are magnetic materials, the attraction force will cause agglomeration and settlement.

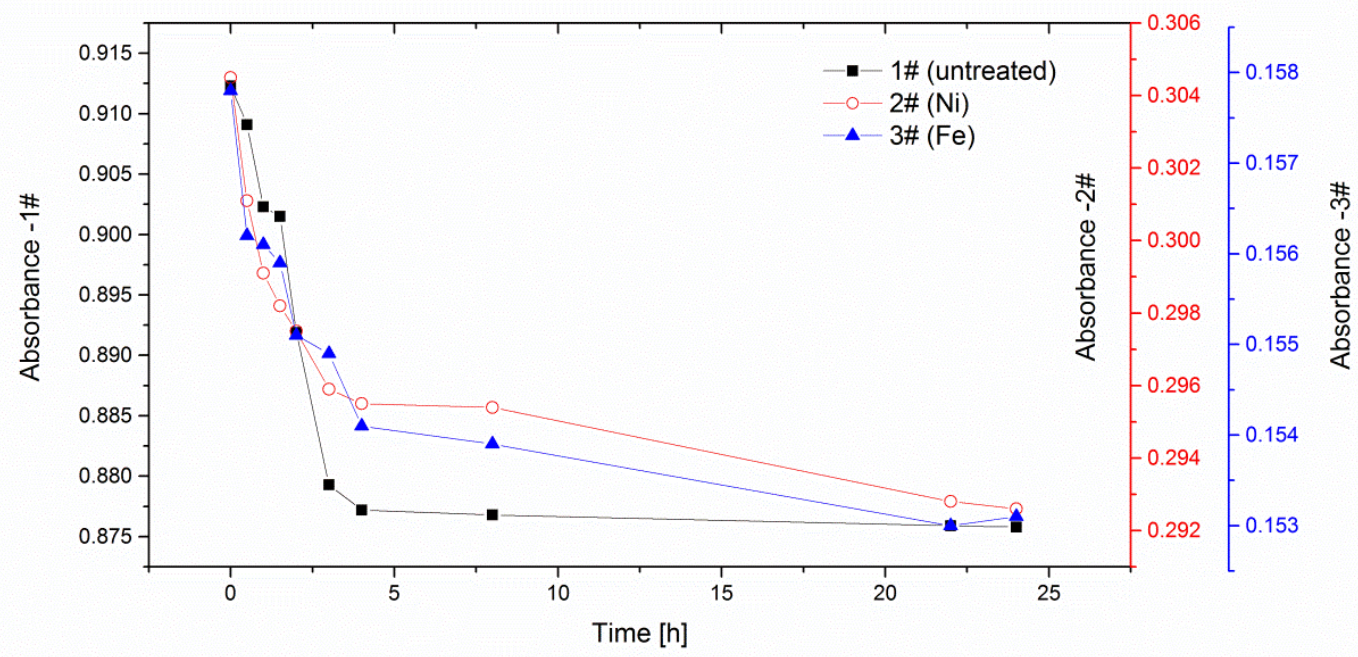

Fig. 1. Relationship between static duration and absorbance

Influence of temperature on dispersion stability. Fig. 2 shows the variation of absorbance of untreated MWNT dispersions under conditions of temperature control with increasing time. It can be observed that the dispersions with temperature control have higher initial absorbance, indicating good dispersion effects. During the 0-2 hour initial period, the absorbance of dispersions prepared under temperature control decreased at a lower speed compared to that prepared without temperature control. Meanwhile, the overall decrease magnitude was also smaller. During the preparation of dispersions, heat energy generated by ultrasound increased the dispersions temperature from 20 to $50^{\circ} \mathrm{C}[19]$. High temperature accelerated molecular motions, causing secondary agglomeration and subsequent settlement. However, due to temperature control, the dispersions kept at a low temperature, and therefore, had a stable dispersity.

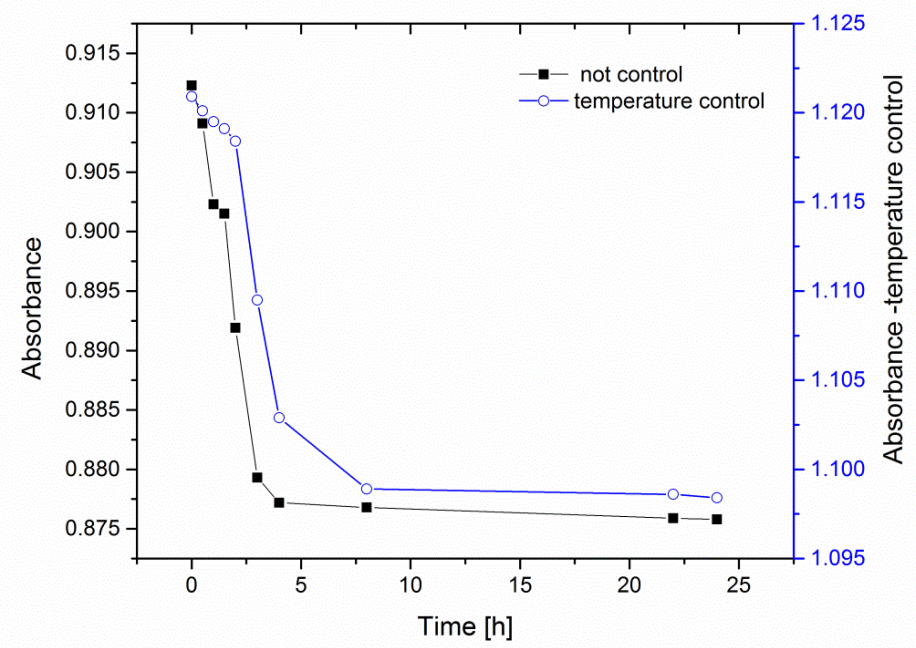

Fig. 2. The influence of temperature control in dispersion process on stability of untreated MWNT dispersions 


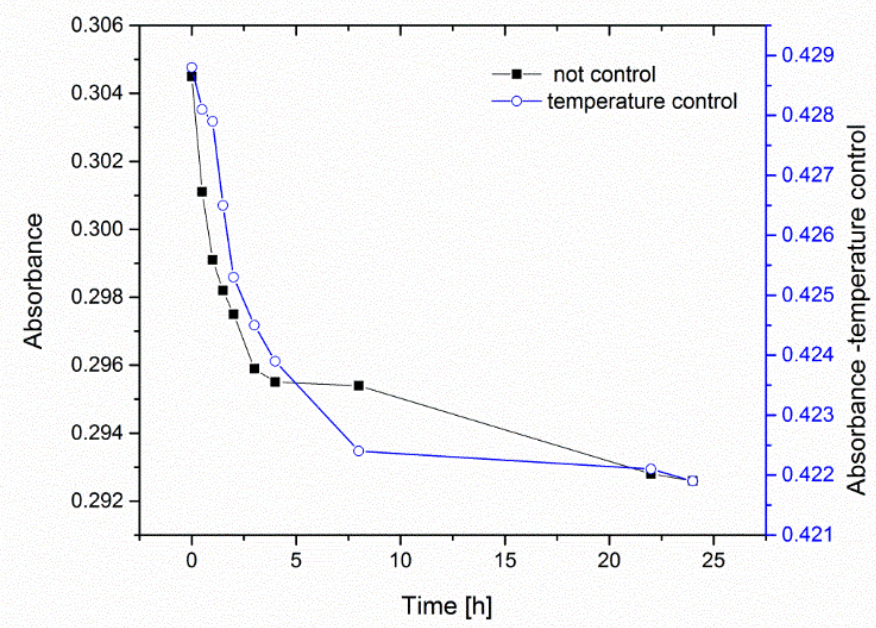

Fig. 3. The influence of temperature control in dispersion process on stability of nickel-coated MWNT dispersions

Fig. 3 shows the variation of absorbance of nickel-coated MWNT dispersions under conditions of temperature control with increasing time. It can be seen, similarly, that in the period of 0-2 hours the absorbance of dispersions prepared under temperature control showed smaller decreasing speed and magnitude compared to that without temperature control. Besides, the dispersions with temperature control reached stable condition earlier than the dispersions prepared without temperature control.

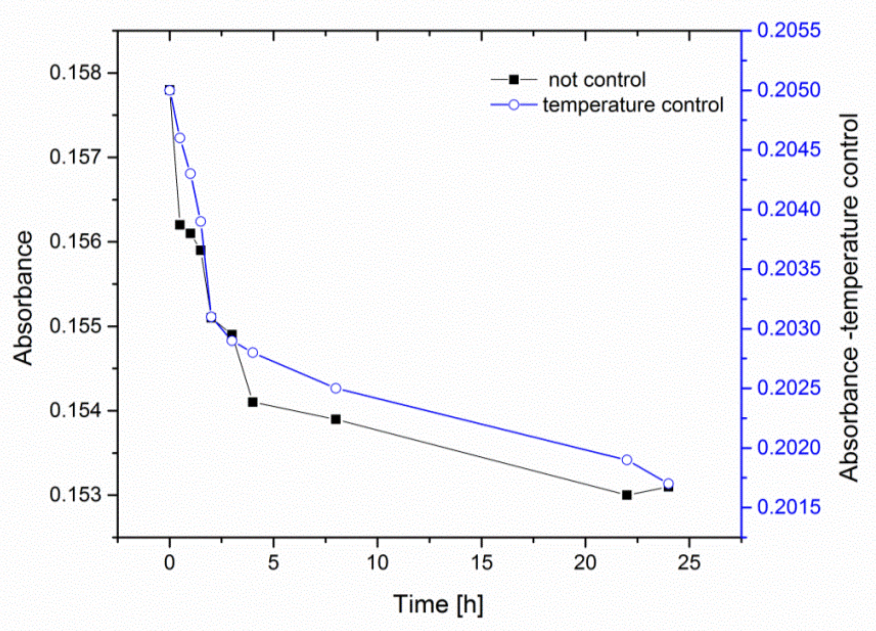

Fig. 4. The influence of temperature control in dispersion process on stability of iron-coated MWNT dispersions

Fig. 4 shows the variation of absorbance of iron-coated MWNT dispersions under conditions of temperature control with increasing time. It can be seen that the dispersions prepared under temperature control demonstrated smaller fluctuation in absorbance than the dispersions prepared without temperature control, as well as smoother decrease during 0-2 hour period, smaller decrease magnitude, and less time before reaching stable stage. The iron particles combined with carbon nanotubes have high temperature sensitivity. Meanwhile, the carbon nanotube can easily break into particles with varying sizes during dispersion process, leading to complex particles patterns. So its dispersity is more sensitive to temperature.

Overall, temperature control played a positive role in dispersity of each type of carbon nanotubes. The decrease of dispersity as a result of molecular motions in the ultrasonic process was effectively eliminated or mitigated, although the degree of mitigation varied among different materials. Additionally, temperature control resulted in a more stable dispersion process, and better dispersion effects. 
Influence of dispersants type on dispersion stability. SDBS, oleic acid, and TENDIS were employed for dispersion of three types of carbon nanotubes. Fig. 5 shows the optimal available time (time before 5\% decrease of absorbance) for three types of carbon nanotubes with each presenting its optimal technical parameters.

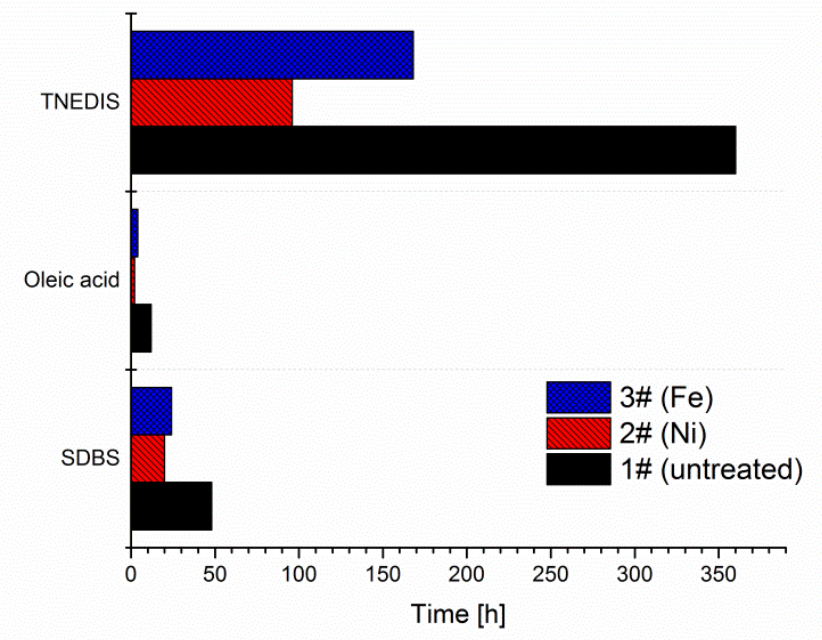

Fig. 5. Available time of dispersions of three types of carbon nanotubes prepared with optimal dispersant technical parameters

It can be seen from Fig. 5 that the carbon nanotubes dispersions prepared with polymer dispersant TNEDIS could keep good dispersion quality in a long period, demonstrating longest available time. Specifically, the available time of TNEDIS dispersions of untreated carbon nanotubes was as long as 360 hours, followed by that of iron-coated carbon nanotubes, and then nickel-coated carbon nanotubes. The carbon nanotubes dispersion prepared with SDBS as dispersant showed shorter available time than TNEDIS dispersions, and the oleic acid dispersions demonstrated the shortest available time. Moreover, the type of carbon nanotube also greatly influenced the available time. The order of available time for three types of carbon nanotubes was untreated carbon nanotubes, iron-coated carbon nanotubes and nickel-coated carbon nanotubes. This is due to the increased density and decreased aperture of carbon nanotubes as a result of metal coating, which could help the settlement. Besides, the metal particles are magnetic materials, the attraction force will cause agglomeration and settlement.

Influence of oscillation on available time. The dispersions were oscillated to a uniform status before using. The influence of oscillation on the available time of carbon nanotubes dispersions is shown in Fig. 6. 


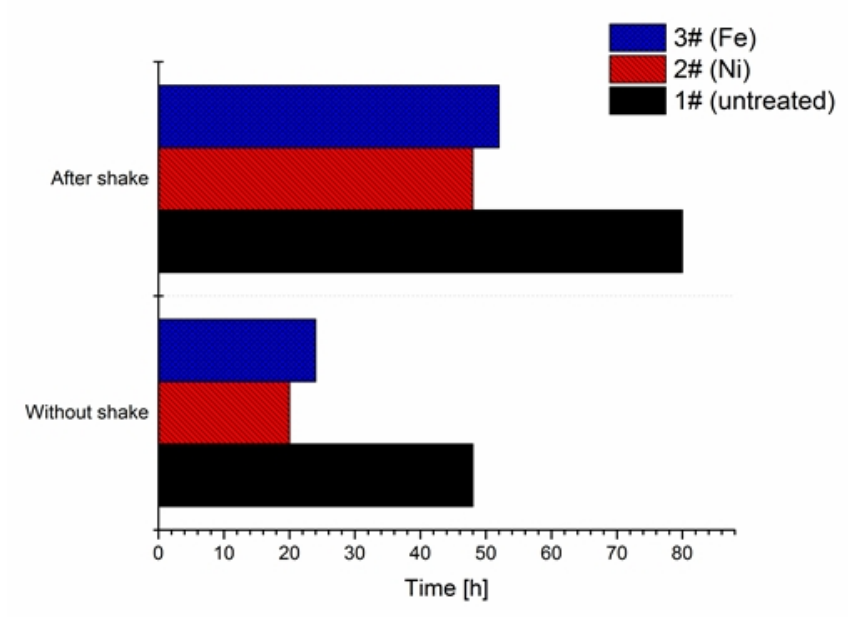

Fig. 6. Influence of oscillation on available time

It can be seen from Fig. 6 that the available time increased after oscillation, which is because of that the soft agglomeration, which accounts for a large part of agglomerations in the dispersions, breaks after the oscillation. This part agglomeration does not have much influence on the carbon nanotubes dispersions.

\section{Conclusions}

In this paper, water bath ultrasonic treatment was employed to prepare dispersion of untreated MWNT, nickel-coated MWNT and iron-coated MWNT. The suspension stability of the prepared dispersions was investigated. The main conclusions are as follows:

(1) Metal coating increased the weight of carbon nanotubes, resulting in difficulties in dispersions during ultrasonic dispersion process. It also accelerates settlement, thereby reducing the stability of the dispersions.

(2) The dispersions prepared under constant temperature condition demonstrated superior dispersity and dispersion stability. The order of sensitivity of dispersity to temperature was untreated carbon nanotubes < nickel-coated carbon nanotubes < iron-coated carbon nanotubes.

(3) The order of dispersion stability was untreated carbon nanotubes > iron-coated carbon nanotubes> nickel-coated carbon nanotubes. The order of dispersants effects was TNEDIS > SDBS > oleic acid.

\section{References}

[1] Y. Yosida. High-temperature shrinkage of single-walled carbon nanotube bundles up to $1600 \mathrm{~K}$. J. Appl. Phys. 87 (2000) 3338-3341.

[2] S. Berber, Y.K. Kwon, D. Toma'nek. Unusually high thermal conductivity of carbon nanotubes. Phys. Rev. Lett. 84 (2000) 4613-4616.

[3] J. H. Du, J. Bai, H.M. Cheng. The present status and key problems of carbon nanotube based polymer composites Express. Polym. Lett.1 (2007) 253-273.

[4] D. A. Heller, R. M. Mayrhofer,S. Baik et al. Concomitant length and diameter separation of single-walled carbon nanotubes. J. Am. Chem. Soc.126 (2004) 14567-14573.

[5] G. Liu, Y. Zhao,K. Zheng et al. Coulomb Explosion: A Novel Approach to Separate Single-Walled Carbon Nanotubes from Their Bundle .Nano. Lett. 9 (2009) 239-244.

[6] T. Tanaka, H. H. Jin,Y. Miyata et al. Simple and Scalable Gel-Based Separation of Metallic and Semiconducting Carbon Nanotubes .Nano. Lett. 9 (2009) 1497-1500. 
[7] M. Horton, H. Hong, C. Li, B. Shi, G. P. Peterson. Magnetic alignment of Ni-coated single wall carbon nanotubes in heat transfer nanofluids. J. Appl. Phys. 107(2010) 104320-1-4

[8] A. L. Elias, J. A. Rodriguez-Manzo, M. R. McCartney et al. Production and characterization of single-crystal FeCo nanowires inside carbon nanotubes. Nano. Lett. 5 (2005) 467-472.

[9] S.J. Yoo, S.H.Han,W.J.Kim. A combination of ball milling and high-ratio differential speed rolling for synthesizing carbon nanotube/copper composites. Carbon. 61 (2013)487-500

[10] K. J. Ziegler, Z. J. Gu, Z. Chen, et al. Cutting single-walled carbon nanotubes. Nano. Techn. 16 (2005) 539

[11] E. C. W. Ou, L. Hu, G. C. R. Raymond, O. K. Soo, J. Pan, Z. Zheng et al.: 'Surface-Modified Nanotube Anodes for High Performance Organic Light-Emitting Diode', ACS Nano. 3 (2009) 2258-2264.

[12] K. Yurekli, C.A. Mitchell, R. Krishnamoorti. Small-angle neutron scattering from surfactant-assisted aqueous dispersions of carbon nanotubes. J. Am. Chem. Soc.126 (2004)9902-9903.

[13] A. Star, J. F. Stoddart, D. Steuerman. Preparation and properties of polymer-wrapped single-walled carbon nanotubes [J]. Angew. Chem. Int. Ed. 40 (2001) 1721

[14] V. Lordi, N. Yao, J. Wei. Method for supporting platinum on single-walled carbon nanotubes for a selective hydrogenation catalyst [J].Chem. Mater. 13(2001)733

[15] K.L. Lu, R.M. Lago, Y.K. Chen, M.L.H. Green, P.J.F. Harris, S.C. Tsang. Mechanical damage of carbon nanotubes by ultrasound. Carbon. 34 (1996) 814-816.

[16] M.D. Rossell, C.Kuebel, G.Ilari, Impact of sonication pretreatment on carbon nanotubes: A transmission electron microscopy study. Carbon. 61(2013) 404-411.

[17] C. Lu, Z. Zhang, J. Zhou, S. Zhang. Influence of Different Dispersants on the Dispersion Stabilities of Nickel Nanopowders in Ethanol. Materials Review. 5 (2007)165-167

[18] C. Wang, W. Liu, J. Li. The study methods of poly dispersant's disperse capability and mechanism. Hebei Chemical Engineering and Industry. 4 (2004)12-16

[19] X. -n. Liang and W. Li, J. Dispers. Sci. Technol. DOI:10.1080/01932691.2015.1101608. 